Jahangirnagar University J. Biol. Sci. 4(2): 9-17, 2015 (December)

\title{
Pattern of antibiotic use and physician's opinion about the resistance against antibiotics used for treating Respiratory Tract Infections (RTIs) in Bangladesh: A cross sectional survey
}

\author{
Kaniz Farjana, Hasan Imam Zahid ${ }^{1}$, M Salahuddin Bhuiya and Saquiba Yesmine* \\ Department of Pharmacy Jahangirnagar University, Savar, Dhaka-1342, Bangladesh
}

\begin{abstract}
Respiratory Tract Infections (RTIs) are major causes of illness, doctor visit and high rates of antibiotic prescriptions in Bangladesh. Over the past decades, emergence of antibiotic resistance has been recognized as an important public health problem across the globe. The current study was aimed to assess the physicians' prescribing pattern of antibiotics in treating RTIs, their usages by patients and physicians' opinion regarding the causes of antimicrobial resistance. This cross sectional survey was conducted by a self-designed standard questionnaire and data were collected manually over a two-month period from November 15 , 2013 to January 18, 2014 in four cities of Bangladesh: Dhaka, Chittagong, Rajshahi and Khulna. According to physicians' opinion, the most frequently prescribed antibiotic for RTIs was levofloxacin (33.44\%) followed by azithromycin (24.12\%), cefixime (18.33\%) and ceftriaxone (17.84\%), respectively. Erythromycin was found to be the most resistant antibiotic $(32.13 \%)$. Two or more antibiotics were prescribed in $43.94 \%$ of prescriptions. A total of $65.15 \%$ prescriptions had complete direction for antibiotics use and $46.97 \%$ patients completed full course of antibiotics. Although $86.36 \%$ prescriptions had no clinical test for using antibiotics, the percentages of patients' disease recovery were $84.85 \%$ and noncompliance was $15.15 \%$. Most of the physicians believed that self-medication of antibiotics results in the spread of bacterial resistance to antibiotics in RTIs. The present findings are important for public awareness and education in antibiotic use. These findings also have significance to the regulations of prescribing antibiotics in RTIs in Bangladesh.
\end{abstract}

Key words: Respiratory Tract Infections, Antibiotic resistance, Bangladesh.

\section{INTRODUCTION}

Respiratory tract infections (RTIs) may be classified into upper respiratory tract infections (URTIs) and lower respiratory tract infections (LRTIs), depending on the main organs affected such as nose, sinuses, middle ear, larynx and pharynx versus trachea, bronchi and lungs. URTIs are usually mild in nature and most often caused by viruses, sometimes with a bacterial component as in some cases of sinusitis and otitis media (Nyquist et al., 1998; Simoes et al., 2006). The majority of RTIs related severe illness and deaths are due to LRTIs, comprising mainly of pneumonia (Rahman \& Rahman, 1997). RTIs are the most common cause for doctor visits and prescription of antibiotics (Snow et al., 2001; Biswas et al., 2014). Nowadays, inappropriate use of antibiotics is a global concern that leads to antibiotic resistance and adverse drug reactions.

\footnotetext{
${ }^{1}$ Novelta Bestway Pharmaceuticals Ltd, Dhanmondi, Dhaka, Bangladesh

*Corresponding author. Email: s.yesmine@juniv.edu
} 
Over the past decade, the emergence of antibiotic resistance has been considered as an important public health problem worldwide. Evidence showed that general practitioners (GPs) prescribed more than $90 \%$ of all antibiotics and about $60 \%$ of these were issued for common respiratory tract infections (RTIs) (Gonzales et al., 2001; Gulliford et al., 2014). Prescriptions of antibiotics for upper respiratory tract infections (URTIs) is very common practice in pediatric patients even though evidence demonstrated that antibiotics had little role to improve outcomes in these patients (Gonzales et al., 2001; 2013). Even some of the bacterial illnesses such as otitis media and sinusitis are usually self-limited, and antibiotic treatment is not essential. Further evidence demonstrated that the proportion of antibiotic use in the community is directly associated to the development of antibiotic resistance. An unnecessary prescription of antibiotics is the main driver for the development of antibiotic resistance and both pediatricians and parents contribute to this problem. The incidence and duration of prior antibiotic exposure are strongly associated with the spread of drug-resistant $S$. pneumoniae, and children receive a significant proportion of the total antibiotics prescribed each year (Nyquist et al., 1998; Holmes et al., 1997).

In a developing country like Bangladesh, the cost of health care is a deciding factor for doctor visit, taking medications and completion of doses. Prescribing excessive medicines and combination therapy substantially increase the total cost of a prescription. The practitioners should be made aware of the importance of combination therapy in the treatment of certain infections so that the chance of resistance development can be ameliorated to the most possible extent. Many studies confirmed that the antibiotics were among the major group of drugs, which were frequently used and caused adverse drug reactions (ADRs) (Padmaja et al., 2009; Remesh et al., 2013). Over the last few decades Bangladesh has made significant progress in drug manufacturing since the promulgation of 'Drug Control Ordinance-1982'. However, irrational use, inappropriate prescribing and self-medication of antibiotics often increase the cost of therapy and contribute to emergence of resistant micro-organisms. Many doctors in Bangladesh are prescribing antibiotics irrationally without taking consideration of the clinical test in most cases. On the other hand, the patients are not completing the total dosage regimen of antibiotics, if it is given in cold and general fever or even in other complicated infectious diseases.

Therefore, this survey based research was aimed to assess: (i) the physicians' prescribing pattern of antibiotics in RTIs, (ii) antibiotics usage by patients and (iii) physicians' opinion regarding the causes of antimicrobial resistance in four cities of Bangladesh.

\section{MATERIALS AND METHODS}

Study design, setting and study population: The present survey based research was a cross-sectional prospective study carried out in the adjacent areas of four district cities of Bangladesh: Dhaka, Chittagong, Rajshahi, and Khulna. Data were collected for over a two- month period from November 15, 2013 to January 18, 2014.

Data collection: This cross-sectional health survey was carried out with a self-designed standard questionnaire by directly interviewing 200 qualified doctors from four district cities, 50 from each city respectively. Prescribing physicians were selected randomly that 
include different groups: resident doctors, GPs, specialists and consultants (Biswas et al., 2014). Prescription data were collected from the patients by random selecting the patients who came to buy the drugs from the pharmacies. The data collectors were waiting in front of the pharmacy shop and convince them to produce their prescription. Prescriptions from the qualified doctors were only analyzed and presented in this paper.

Ethical Considerations: The research was conducted following the general principles (section 12) of WMA declaration of Helsinki. This survey based research was logistically supported by the Department of Pharmacy, Jahangirnagar University and Novelta Bestway Pharmaceuticals Ltd. As the human subjects involved in our study only participated in the interview, this survey based research did not take any further approval from institutional ethics committee.

Statistical Analysis: Data were analyzed using Microsoft Excel 2007. Data were presented as numbers, percentages and proportions. Outputs were presented in both graphs and tables. The data were subjected to simple descriptive statistical analyses including frequency distribution, mean and percentage.

\section{RESULTS AND DISCUSSION}

Based on the collected prescriptions, the respiratory tract infections were categorized according to the age groups and sex. Results showed that the incidence of RTIs were highest $(46.97 \%)$ among the school age populations of 5-15 years old and among the elderly populations of $>60$ years old for both sexes (Table 1). The results of this study indicated that highest number of physicians (33.44\%) preferred to prescribe levofloxacina fluoroquinolone antibiotic followed by azithromycin which was favoured by $24.12 \%$ physicians in treating RTIs (Figure 1). Other preferred antibiotics were cefixime (18.33\%) and ceftriaxone (17.84\%) (Figure 1). The findings of our prescription survey (Figure 1) also showed that levofloxacin was the most prescribed antibiotic $(39.39 \%)$ followed by azithromycin (34.85\%) and cefixime (13.64\%), respectively (Figure 1). Although 1.23\% physicians voted amoxicillin as the preferred antibiotic, we did not get a single prescription for amoxicillin (Figure 1)

Table 1. Age and sex distribution of collected prescriptions for cases of Respiratory Tract Infections (RTIs)

\begin{tabular}{c|cc|cc|c|c}
\hline $\begin{array}{l}\text { Age Group } \\
\text { (in Year) }\end{array}$ & \multicolumn{2}{c|}{$\begin{array}{c}\text { Frequency of } \\
\text { Prescription (TNP=66) }\end{array}$} & \multicolumn{2}{l|}{ Individual Percentage (\%) } & $\begin{array}{c}\text { Total } \\
\text { Frequency }\end{array}$ & AVP (\%) \\
\hline $5-15$ & Male & Female & Male & Female & & \\
$15-30$ & 18 & 13 & 46.15 & 48.15 & 31 & 46.97 \\
$31-45$ & 3 & 2 & 7.69 & 7.41 & 5 & 7.58 \\
$46-60$ & 2 & 1 & 5.13 & 3.70 & 3 & 4.55 \\
$>60$ & 5 & 3 & 12.82 & 11.11 & 8 & 12.12 \\
Total & 11 & 8 & 28.21 & 29.63 & 19 & 28.79 \\
\hline
\end{tabular}

TNP=Total Number of Prescriptions, AVP=Average Percentage 


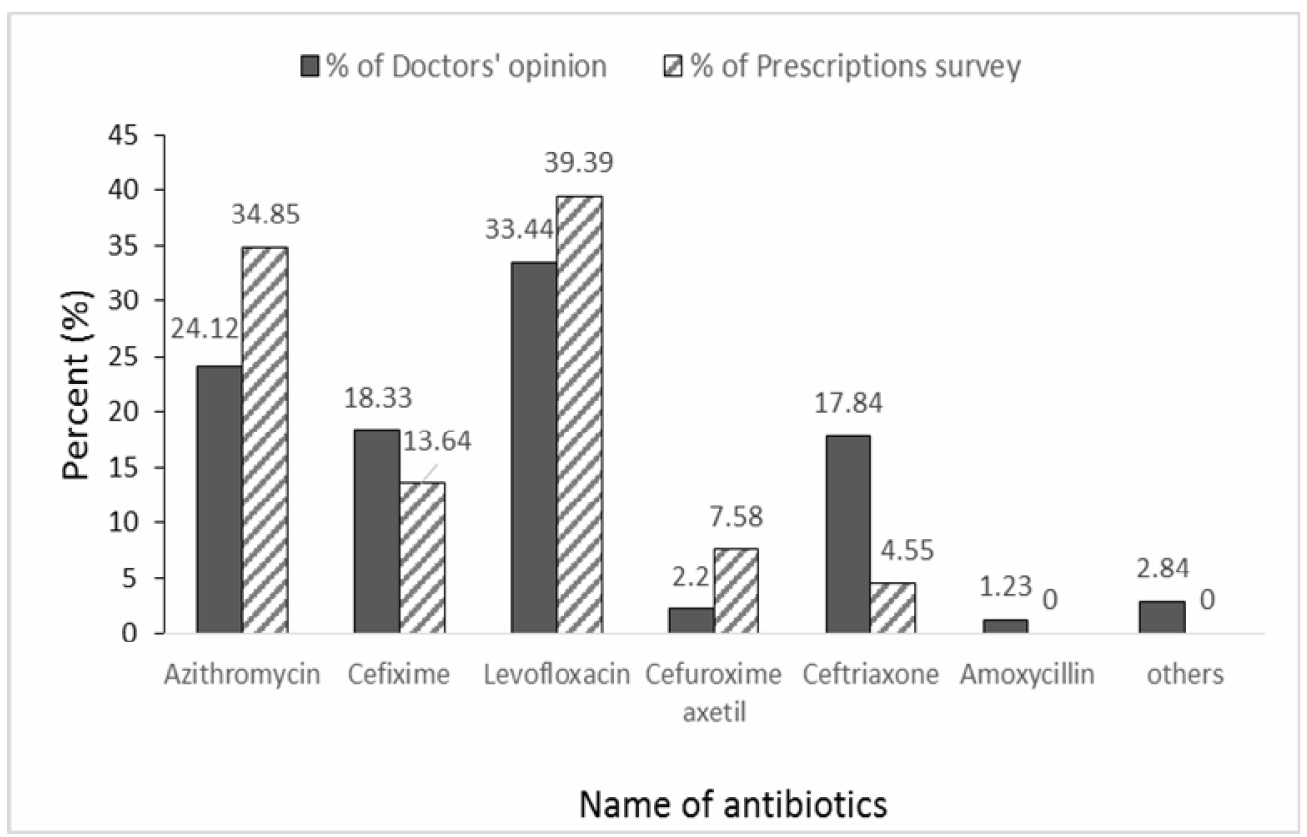

TMP-SMX: Trimethoprim-sulfamethoxazole

Fig. 1. Pattern of antibiotic prescription in RTIs according to doctors' opinion and prescriptions survey

This study showed that according to doctors' opinion, erythromycin (32.13\%), amoxicillin (30.14\%) and trimethoprim-sulfamethoxazole (25.34\%) were considered as the most resistant antibiotics in RTIs (Figure 2).

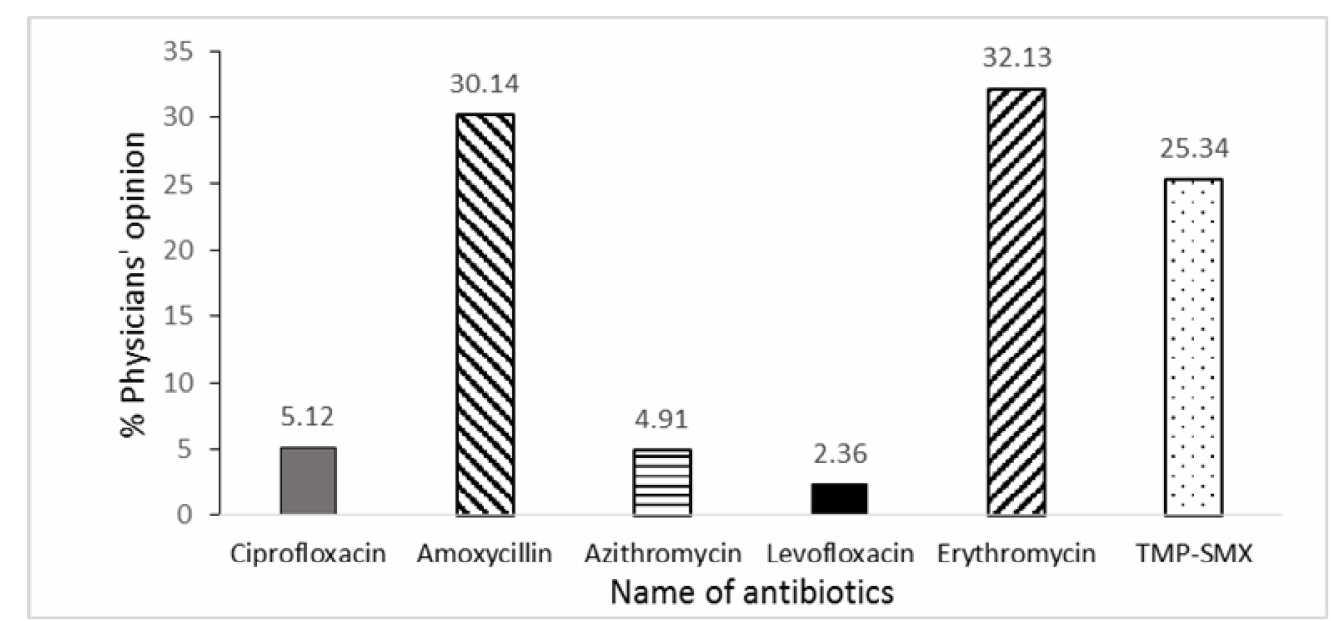

Fig. 2. Scenario of resistant antibiotics in RTIs according to doctors' opinion 
In this study, $36.43 \%$ of physicians said that self-medication was a major contributing factor to drug resistance and whereas $32.5 \%$ of physicians considered incomplete dose as the reasons for drug-resistance (Figure 3). A total 14.43\% of physicians answered overconsumption of antibiotics which is mentioned as 'overuse' in the Figure 3 and $16.64 \%$ of physicians mentioned inadequate dose and duration are responsible for antibiotic resistance in Bangladesh (Figure 3).

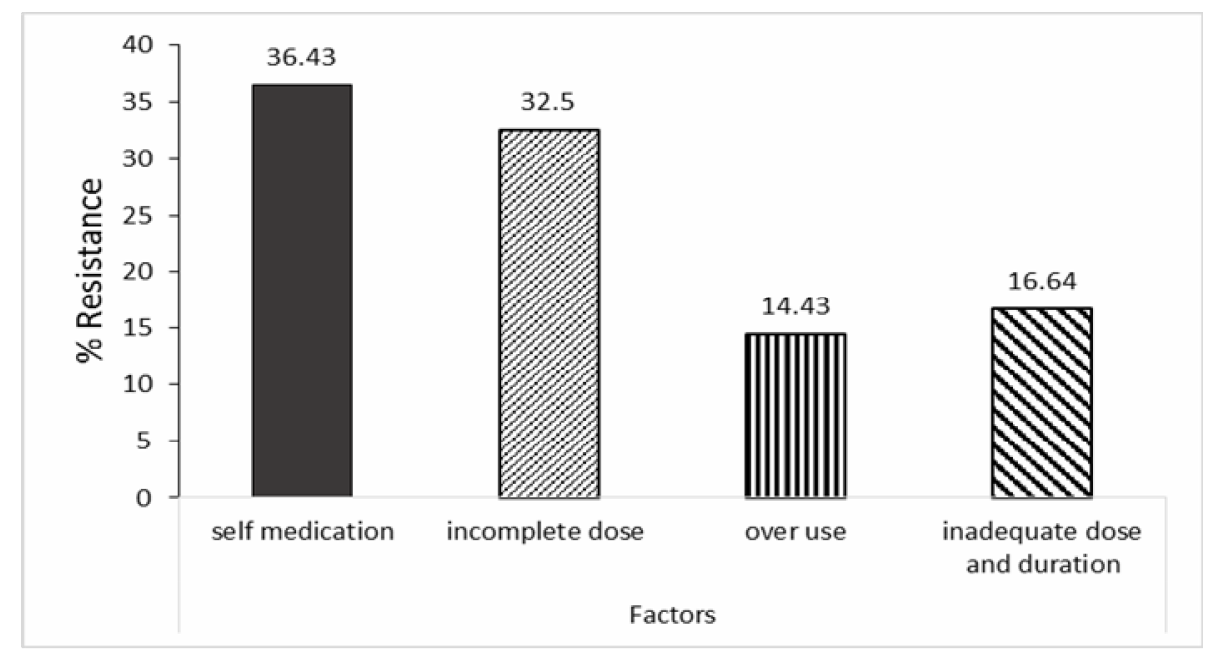

Fig. 3. Causes of antibiotic resistance in RTIs according to doctors' opinion

In this study, two or more antibiotics were found to be prescribed in $43.94 \%$ of prescriptions (Table 2). A complete direction for antibiotics use was given in $65.15 \%$ of prescriptions and $46.97 \%$ patients completed full course of antibiotics (Table 2). Although $86.36 \%$ prescriptions had no clinical test for using antibiotics, the percentages of patients' disease recovery was $84.85 \%$ (Table 2). However, a considerable percentage of patients (15.15\%) showed noncompliance to antibiotics (Table 2).

Table 2. Antibiotic prescription and usages against different parameters of RTI patients in Bangladesh

\begin{tabular}{l|l|c|c}
\hline Parameters & Prescription Pattern & $\begin{array}{c}\text { Frequency } \\
(\mathrm{TNP}=66)\end{array}$ & $\begin{array}{c}\text { Percentage } \\
(\%)\end{array}$ \\
\hline Pattern of antibiotics Prescription & Single antibiotic & 37 & 56.06 \\
& Multiple antibiotics & 29 & 43.94 \\
Information on direction for & Complete & 43 & 65.15 \\
antibiotic use & Not mentioned & 23 & 34.85 \\
Clinical test for prescribing & With test & 9 & 13.64 \\
antibiotics & Without test & 57 & 86.36 \\
Completion of full antibiotic course & Yes & 31 & 46.97 \\
& No & 35 & 53.03 \\
Patient's compliance & Disease recovery & 56 & 84.85 \\
& Noncompliance & 10 & 15.15 \\
\hline
\end{tabular}

TNP=Total Number of Prescriptions 
The present study provides a brief scenario of the present status of RTI in Bangladesh and prescription pattern of antibiotics in treating RTIs and major reasons behind antibiotic resistance in Bangladesh.

Results from our prescription survey showed that incidence of RTIs was highest among children of $<15$ years of age and among the geriatric patients. This result (Table 1) corroborated with a study conducted by Biswas et al. 2014 and indicated that children and geriatric populations perhaps are more prone to infections (Biswas et al., 2014). A previous study also showed that prevalence of RTIs was much higher in children under 5 years of age than other age groups in Africa (Yousif \& Khaleq, 2006). The children aged $<5$ years were more prone to infection may be due to a lack of immunity transferred through mother's milk. Another study found that low socioeconomic status, illiteracy, poor nutritional status, overcrowding, indoor air pollution and parental smoking behavior were the significant social and demographic risk factors responsible for RTIs in underfive children (Goel et al., 2012). Increasing female literacy level and health education might contribute in prevention of morbidity and mortality amongst children in RTIs.

In the prescription survey, the prevalence of respiratory tract infections was found to be slightly higher in male $(59.09 \%)$ than in female $(40.90 \%)$ (Table 1). Regarding the first line choice of antibiotics in cases of RTIs, the present survey showed that the majority of the physicians preferred levofloxacin followed by azithromycin or cefixime. This is perhaps because levofloxacin was indicated by the US Food and Drug Administration (FDA) for penicillin-resistant S. pneumoniae where ceftriaxone and azithromycin were not recommended (Zafar et al., 2008, Holmes et al., 1997). Several in vitro studies showed that levofloxacin worked better to treat RTIs where major pathogens are gramnegative bacteria because of its broad spectrum gram negative coverage (Snow et al., 2001). However, third generation ceftriaxone is a broad spectrum antibiotic and has been recommended for the treatment in RTIs and several physicians have claimed good results with them, particularly with cefixime (Zafar et al. 2008). Patients of acute RTIs are managed with a wide of range of antibiotics, third generation oral cefixime may improve the effectiveness of the treatment due to their strong beta lactamase stability against respiratory pathogens (Zafar et al., 2008).

Results of the present study showed that the majority of doctors expressed their opinion that self-medication, incomplete dose and duration, over-consumption of antibiotics are the main reasons for developing drug resistance. Other studies showed that overprescribing and overuse of medicine are frequently seen in public and private hospitals, clinics and pharmacies (Akkerman et al., 2005; Gonzales et al., 2001). Evidence showed that 45 to 80 percent of patients with symptoms of upper respiratory tract infections and diarrhoea received antibiotic, even though it might not be beneficial if they have a viral illness rather than a bacterial one (Akkerman et al., 2005; Gonzales et al., 2001) .

In a developing country like Bangladesh, majority of the health professionals are not qualified and they do not prescribe antibiotics by following the prescription guidelines of 
antibiotics. Evidence showed that antibiotics are the most frequently prescribed and misused drugs by patients and prescribers (Remesh et al., 2013). Although the maximum antibiotics were prescribed for patients in Bangladesh without clinical test (Table 2) and a considerable number of prescription were without having complete direction for antibiotic use (Table 2), however the percentage of patients' disease recovery was positive (Table 2). This result might be due to physicians' long clinical experience and as well as of broad spectrum nature of the prescribed antibiotics. In Bangladesh, most of the people do not have the minimal awareness regarding resistance, antibiotics and infections. In this study, the majority of doctors expressed their opinion that irrational self-medication, incomplete dose and inadequate duration of antibiotics are the major causes for development of drug resistance.

According to the doctors' opinion, erythromycin and amoxicillin were not found to be potential options for the treatment of respiratory tract infections perhaps due to an increasing trend of developing resistance against these antibiotics (Zafar et al., 2008; Gulliford et al., 2014). Evidence from previous studies demonstrated increasing resistance of erythromycin, amoxicillin and trimethoprim-sulfamethoxazole against pathogens of respiratory infections (Zafar et al., 2008; Gulliford et al., 2014). Nowadays, genifloxacin and clavunalic acid are used to treat respiratory tract infection.

It was also found that doctors prescribe macrolides in various specialized cases. Antibiotic macrolides are used to treat infections caused by gram-positive bacteria (e.g., Streptococcus pneumoniae) and Haemophilus influenzae infections such as respiratory tract and soft-tissue infections.

According to doctors' opinion, the major contributing factors to drug resistance were selfmedication, incomplete dose and duration and over-consumption of medicines. The result of this survey indicated that the general population has a tendency to prematurely discontinue antibiotic regimens. Therefore, when they self medicate antibiotics, they discontinue drugs after feeling better.

Another study showed that the misuse incidences are mostly common for self medicating with ciprofloxacin to treat menstrual irregularity and abdominal pain, amoxicillin to treat viral diseases, ampicillin to treat scabies, azithromycin to treat pain and many others (Butler et al., 2012; Gonzales et al., 2013; Little et al., 2013). Moreover, increased population can lead to increased dissemination of antibiotic resistant bacteria and resistant genes. As Bangladesh is a developing country, increase in population density and urbanization may facilitate the spread of resistant bacteria and gene, which was also seen in other countries. Inconclusive diagnosis and over dependence on antibiotics can be causes for this and environmental variance. In addition to increasing antimicrobial drug resistance, the excess use of antibiotics can lead to unnecessary side effects and increase future consultations for RTIs (Butler et al., 2012). A number of trials have now shown that antibiotic prescribing may be reduced through educational interventions, together with feedback of prescribing information (Butler et al., 2012; Gonzales et al., 2013; Little et al., 2013). 
In brief, this survey based research showed that children and elderly populations were mainly susceptible to respiratory tract infections, multiple antibiotics were prescribed in many cases and antibiotic courses were not completed in substantial number of cases of RTIs. The findings of our study will help to put into developing strategies to reduce overconsumption and to ensure rational use of antibiotics for RTIs in general practice. We need to formulate active professional guidelines concerning an overall level of antibiotic utilization for RTIs that might be acceptable. Results of this study have significant implication for implementing these guidelines for rational use of antibiotics in RTIs.

Conflict of Interest: The authors declare that the article content has no conflict of interest.

Acknowledgment: The authors would like to thank Novelta Bestway Pharmeceuticals Ltd. and Department of Pharmacy Jahangirnagar University for giving logistic support to conduct this survey based research.

\section{REFERENCES}

Akkerman, A.E., Kuyvenhoven, M.M., van der Wouden, J.C., Verheij, T.J.M. 2005. Analysis of under- and overprescribing of antibiotics in acute otitis media in general practice. $J$ Antimicrob Chemother;; doi:10.1093/jac/dki257; [published online 20 July

Award, A.J. and Himad, H.A. 2006. Drug use practices in the teaching hospitals of Khartoum state, Sudan. Eur J Clin Pharmacol. 62:1087-93.

Biswas, M., Roy, D.N., Tajmim, A., Rajib, S.S., Hossain, M., Farzana, F. and Yasmen, N. 2014. Prescription antibiotics for outpatients in Bangladesh: a cross-sectional health survey conducted in three cities. Ann Clin Microbiol Antimicrob. 13:15-22.

Butler, C.C., Simpson, S.A., Dunstan, F., Rollnick, S., Cohen, D., Gillespie, D., Evans, M.R., Alam, M.F., Bekkers, M.J., Evans, J., Moore, L., Howe, R., Hayes, J., Hare, M., Hood, K. 2012. Effectiveness of multifaceted educational programme to reduce antibiotic dispensing in primary care: practice based randomised controlled trial. BMJ. 344:d8173.

Goel, K., Ahmad, S., Agarwal, G., Goel, P., Kumar, V. 2012. A cross sectional study on prevalence of acute respiratory infections (ARI) in under-five children of Meerut district, India. J Com Med \& Health Edu.2:176. doi: 10.4172/2161-0711.1000176.

Gonzales, R., Anderer, T., McCuloch, C.E., Maselli, J.H., Bloom, F.J.Jr., Graf, T.R., Shah,.M., Yefko, M., Molecavage, J., Metlay, J.P. 2013. A cluster randomized trial of decision support strategies for reducing antibiotic use in acute bronchitis. JAMA Intern Med. 173:267-73.

Gonzales, R., Daniel, C., Malone, Judith, H. Maselli, Merle A. Sande. 2001. Excessive antibiotic use for acute respiratory infections in the United States. Clinical Infectious Diseases. 33:757-62.

Gulliford, M.C., Dregan, A., Moore, M.V., Ashworth, M., van Staa, T., McCann, G., Chariton, J., Yardley, L., Little, P., McDermott, L. 2014. Continued high rates of antibiotic prescribing to adults with respiratory tract infection: survey of $568 \mathrm{UK}$ general practice; $B M J$ Open.4:10. e006245 doi:10.1136/bmjopen-2014-006245.

Holmes, S.J., Solomon, S.L., Morrow, A.L., Schwartz, B. and Pickering, L.K. 1997. Risk factors for carriage of penicillin-resistant Streptococcus pneumoniae (S. pneumoniae) in young children. Pediatr Res.41:122A. 
Little, P., Stuart, B., Francis, N., Douglas, E., Tonkin-Crine, S., Anthierens, S., Cals, J.W., Melbye, H., Santer, M., Moore, M., Coenen, S., Butler, C., Hood, K., Kelly, M., Godycki-Cwirko, M., Mierzecki, A., Torres, A., Llor, C., Davies, M., Mullee, M., O'Reilly, G., van der Velden, A., Geraghty, A.W., Goossens, H., Verheij, T., Yardley, L. 2013. Effects of internet-based training on antibiotic prescribing rates for acute respiratory-tract infections: a multinational, cluster, randomised, factorial, controlled trial. Lancet.382:1175-82. doi: 10.1016/S0140-6736(13)60994-0. Epub 2013 Jul 31.

Nyquist, A.C., Gonzales, R., Steiner, J.F. and Sande, M. 1998. Antibiotic prescribing for children with colds, upper respiratory infections and bronchitis by ambulatory physicians in the United States. JAMA. 279:875-7.

Padmaja, U., Adhikari, P. and Pereira, P. 2009. A prospective analysis of adverse drug reactions in a South Indian hospital. Online J Health Allied Sci. 8:12.

Rahman, M.M. and Rahman, A.M. 1997. Prevalence of acute respiratory tract infection and its risk factors in under five children. Bangladesh Med Res Counc Bull. 23: 47-50.

Remesh, A., Samna Salim, A.M., Gayathri, U.N. and Retnavally KG. 2013. Antibiotics prescribing pattern in the in-patient departments of a tertiary care hospital. Pharma Pract. 4:71-76.

Simoes, E.A.F., Cherian, T., Chow, J., Shahid-Salles, A.S., Laxminarayan, R., John, J.T. 2006. Acute respiratory infections in children. In: Jamison DT, Breman J.G., Measham A.R. Alleyne, G., Claeson, M., Evans, D.B., Jha, P., Mills, A., Musgrove, P., editors. Disease Control Priorities in Developing Countries. 2nd edition. Washington (DC): World Bank; 2006. Chapter 25. Available from: http://www.ncbi.nlm.nih.gov/books/NBK11786/.

Snow, V., Mottor-Pilson, C. and Gonzales, R. 2001. Principles of appropriate antibiotic use for nonspecific upper respiratory tract infections in adults. Ann Intern Med 2001. 134:487-489

Yousif, T.K. and Khaleq, B.A. 2006. Epidemiology of acute respiratory tract infections (ARI) among children under five years old attending Tikrit general teaching hospital. Middle East Journal of Family Medicine. 4(3): 48-54.

Zafar, A., Hussain, Z., Lomama, E., Sibille, S. and Irfan, S. 2008. Antibiotic susceptibility of pathogens isolated from patients with community-acquired respiratory tract infections in Pakistan-The active study. Journal of Ayub Medical College. 20(1), 7-9. 